\title{
Compte rendu de séminaire de lecture : "diasporas"
}

\section{Francesco Ragazzi}

\section{(2) OpenEdition}

\section{Journals}

\section{Édition électronique}

URL : http://journals.openedition.org/conflits/1765

DOI : 10.4000/conflits. 1765

ISSN : 1777-5345

Éditeur :

CCLS - Centre d'études sur les conflits lilberté et sécurité, L'Harmattan

\section{Édition imprimée}

Date de publication : 1 mars 2005

Pagination : 251-254

ISBN : 2-7475-8103-9

ISSN : 1157-996X

Référence électronique

Francesco Ragazzi, «Compte rendu de séminaire de lecture : "diasporas" », Cultures \& Conflits [En ligne], 57 | printemps 2005, mis en ligne le 02 juin 2005, consulté le 30 mars 2021. URL : http:// journals.openedition.org/conflits/1765; DOI : https://doi.org/10.4000/conflits.1765

Ce document a été généré automatiquement le 30 mars 2021.

Creative Commons License 


\title{
Compte rendu de séminaire de lecture : "diasporas"
}

\author{
Francesco Ragazzi
}

1 C'est dans le cadre des séminaires de lectures de la revue Cultures \& Conflits qu'a eu lieu la présentation de l'ouvrage Les Diasporas de Stéphane Dufoix, paru dans la collection «Que-SaisJe »(PUF). L'exposé de Stéphane Dufoix a été suivi d'un échange de questions avec la salle'.

Exposé

2 Stéphane Dufoix commence son exposé en soulignant l'importance de faire l'histoire des mots, ce qui constitue une partie de son programme de recherche actuelle, dédiée à la généalogie de la notion de Diaspora.

Généalogie.

4 Si le terme de Diaspora fait effectivement son apparition dans la Bible des Septante, il faut se démarquer de l'interprétation biaisée - et reprise par de nombreux auteurs qu'a fait Robin Cohen (auteur de Global Diasporas) de l'apparition du mot. En effet, le mot n'apparaît jamais comme une traduction des termes hébreux de Galut, mais bien comme un néologisme pour désigner la volonté divine, et non humaine, de dispersion du peuple élu. En ce sens, les premières utilisations du terme "diaspora », strictement confinées à un champ lexical religieux, ne se réfèrent absolument pas à la condition physique du peuple juif en dispersion. Successivement, aussi bien dans la littérature Grecque que dans la littérature Latine, le terme de "diaspora " continue à revêtir un aspect essentiellement religieux, et ne sort que très rarement de ce cadre. L'utilisation restrictive du terme de diaspora reste inchangé a peu près jusqu'au début du $\mathrm{XX}^{\mathrm{ème}}$ siècle.

5 A partir de ce moment, pour reprendre les mots de Dufoix, «(...) l'usage actuel de 'diaspora' ne s'appuie ni sur le sens original de diaspeirô (disperser, répartir, distribuer) ni sur la signification de diaspora dans la Bible des Septante, ni encore sur son éventuel usage commun ultérieur en grec, mais bien sur l'interprétation qui en a été faite par les auteurs juifs puis chrétiens (catholiques puis protestants), et dans laquelle est accompli un travail d'homologie entre la dispersion dans le royaume terrestre par rapport au royaume de Dieu et la dispersion loin d'une terre ou d'un centre politico-religieux $»^{2}$. 
Sécularisation, banalisation, formalisation.

6 Selon Dufoix, trois processus vont alors se mettre en place, avec pour conséquence une extension et une diffusion du mot. Tout d'abord, un processus de sécularisation : le mot sort peu à peu du champ exclusivement sacré pour désigner progressivement l'état des populations dispersées en dehors d'une signification religieuse. Second processus, le mot se banalise, que cela soit à travers le discours académique, ou à travers le discours journalistique; le mot se diffuse, et passe ainsi d'un nom propre, à un «semi-nom propre $»^{3}$ (J.C. Passeron). Enfin, dernière étape, celle de l'entrée du terme dans les sciences sociales et sa formalisation comme catégorie archétypique, individuable en fonction d'un nombre déterminé de critères. Ce triple processus, selon Dufoix, ne doit cependant pas être compris comme une succession d'étapes, mais au contraire comme des processus stratifiés interdépendants, de sorte que le discours universitaire informe le discours académique, qui est lui-même modifié et porté à épouser certaines formes du premier.

Mondialisation, transnationalisme et post-modernisme.

7 Dans le champ académique, Dufoix remarque que le terme de "diaspora" semble correspondre parfaitement à la fois au sujet et aux questions théoriques et empiriques posées par trois «nouvelles » tendances dans le monde universitaire des années 1990, à savoir le discours académique sur la mondialisation, le discours en sciences sociales du " transnationalisme » et enfin le courant postmoderne (philosophie) ou culturaliste (anthropologie). Les réflexions autour des d'identités, des migrations, des attachements multiples et du nationalisme à distance semblent trouver dans le terme de diaspora à la fois un outil conceptuel et un slogan efficace, un "argument de distinction, de modernité scientifique et de vente $»^{4}$. La présentation de Stéphane Dufoix s'est enfin conclue sur une revue des questions abordées dans son ouvrage, et plus particulièrement la question des utilisations gouvernementales du terme. Dufoix remarque en effet, en prenant quelques exemples, comme ceux d'Israël, de l'Irlande, de la Russie ou encore de la Croatie, que de plus en plus de gouvernements trouvent dans le terme un moyen efficace pour désigner des "nationaux » à l'étranger dans un contexte de post-Guerre Froide où la distinction entre émigration politique et économique ou temporaire (gastarbieter) perd son sens comme stratégie d'objectivation. Le terme de Diaspora semble alors permettre aux gouvernements de se référer à un ensemble homogène en apparence pour développer des politiques "d'attention», signes d'une « transnationalisation » ou d'un « débordement de l'Etat » qui reste encore à explorer. Discussion

8 La discussion a porté aussi bien sur des points abordés dans le livre que sur des points de la présentation. Le débat s'est ouvert autour de plusieurs questions, principalement sur l'usage des idéaux-types dans le livre, sur l'analyse sans doute manquante (essentiellement due au format du livre) en termes "d'énoncés performatifs », et de mise en lumière des différents énonciateurs du «speech act» à l'œuvre dans la dénomination des diasporas. La question de la spécificité de la construction diasporique par rapport à la construction nationale, ainsi que la question des raisons de l'apparition et de la diffusion du modèle diasporique à partir du début $\mathrm{du} \mathrm{XX}^{\mathrm{ème}}$ siècle ont également fait partie des échanges entre Stéphane Dufoix et la salle.

Histoire du mot et processus sociaux.

9 Un ensemble de questions a porté sur la nature du programme de recherche que Stéphane Dufoix a esquissé au début de sa présentation, c'est-à-dire d'une part 
l'évolution du mot, de ses usages, et d'autre part le changement du rapport politique au territoire et les modifications de l'exercice du pouvoir sur le territoire. Pour Dufoix, ces deux ensembles se recoupent mais ne sont pas en adéquation, d'où les deux directions de recherche. Certaines questions ont porté sur la pertinence de cette distinction, en s'interrogeant sur la validité d'une approche dissociant l'étude du nom des processus sociaux à l'œuvre. Cela a été l'occasion pour Stéphane Dufoix de préciser son point de départ et de souligner l'importance qu'il accordait à mener d'un même mouvement cette recherche.

Etats et Diasporas.

10 En ce qui concerne notamment la question de la transnationalisation de l'Etat, Didier Bigo a souligné le danger d'aborder la question du «débordement » en suivant une métaphore des fluides, qui risque, au bout du raisonnement, de justifier certains discours de la gouvernementalité, et notamment de la "gestion ». Une autre question a porté sur la distinction qui pouvait exister entre " réfugiés », " exilés » et " diaspora », à la fois dans les discours et dans les pratiques. Dufoix, qui a travaillé dans le cadre de sa thèse sur la question de l'exil, a mis en lumière les stratégies différenciées qui pouvaient être liées au choix des termes d'« exilé » et de " diaspora ».

\section{NOTES}

1.. Ce séminaire s'est tenu le lundi 29 mai 2004 au CERI en présence de l'auteur Stéphane Dufoix, présidé par Nathalie Bayon, et discuté par Francesco Ragazzi. 2.. Dufoix S., « De 'Diaspora' à 'diasporas'. La dynamique d'un nom propre », intervention à l'université de Paris I, le 6 mars 2004. Texte disponible sur : http:// histoire-sociale.univ-paris1.fr/Sem/Dufoix-paris1.pdf

3.. Passeron J.-C., Le raisonnement sociologique, l'espace non-poppérien du raisonnement naturel, Paris, Nathan, pp. 60-63.

4.. Ibid.

INDEX

Mots-clés : Diaspora 
AUTEUR

FRANCESCO RAGAZZI

Francesco RAGAZZI est doctorant à l'IEP de Paris en Sociologie des relations internationales. 